\title{
Shape-slant invariance as tested by computer-generated perspective figures by children
}

\author{
NAOYUKI OSAKA \\ Otemon-Gakuin University, Ibaraki, Osaka 567, Japan \\ and \\ MARIKO OSAKA \\ Kyoto University, Kyoto 606, Japan
}

\begin{abstract}
The shape-slant invariance suggests that explicit judgments of shape and slant should be highly correlated. Furthermore, it is likely that shape constancy is based upon the ability to take the slant of the object into account. The hypothesis was tested for children using computergenerated dynamic-surface slant sequences representing a rectangle rotating about a horizontal axis. It was found that as age and texture-density cues increased, percentage of perceived depth response increased. This indicates that depth perception through rotation develops as a function of age. However, 3-year-old children's inability to specify the slant or inability to relate the slant with its shape was found. This suggests that shape-slant invariance is likely to develop as a function of age.
\end{abstract}

Shape constancy has been found in infants 6-8 weeks old. Further, it appears plausible to suppose that shape constancy is based upon taking the slant of the object into account. The study of slant perception of a rotating object has been closely tied to the study of shape perception in which there is an attempt to correlate judgments of shape with judgments of slant, as a test of the shape-slant invariance hypothesis: The shape-slant invariance suggests that explicit judgments of shape and slant should be highly correlated. Bower (1966) studied 1.6- to 2-month-old infants by operantly conditioning a head-turning response to a rectangle slanted at $45 \mathrm{deg}$. $\mathrm{He}$ found that the rectangle at a different slant angle resulted in a large number of responses being made in testing. When a trapezoid was presented at $45 \mathrm{deg}$, infants responded the least. Rosinski and Levine (1976), using a rear-projection technique, investigated elementary school children's ability to perceive slant solely on the basis of texture gradient information. They found that the information provided by a linear perspective was considerably more useful.

How is the slant of a surface perceived, and what kind of cues are available to children to specify slant and rotation? The investigation of slant perception is

Portions of this paper were presented at the annual meeting of the Japan Educational Psychological Association, Tokyo, 1978. The authors are indebted to the teachers and staff of the Rakuto Kindergarten, Kyoto, for their assistance. This study was supported in part by Grant 371038 from the Japanese Ministry of Education. Requests for reprints should be sent to Naoyuki Osaka, Department of Psychology, Otemon-Gakuin University, Ibaraki, Osaka 567, Japan. interesting because the kinds of cues that specify the slant of a surface are also critical in depth perception (Rosinski, 1977). Although adults' perception of slant is quite accurate, there is little experimental evidence about its development. Further, little study has been done in a developmental study on the perception of rotary motion (dynamic slant) for computer-generated displays simulating polar projections.

If a rectangle is in a plane slanted with respect to the line of sight, the image will be that of a trapezoid. The trapezoid in Figure 1 (Panel B, 10-deg condition), according to the invariance hypothesis, may be seen as either (1) a trapezoid in the frontal plane or (2) a rectangle with the top slanted away from the observer (perceived depth). If children cannot take the slant into account for shape judgments, they are likely to fail an attempt to correlate judgments of slant with judgments of shape, thus resulting in a flat trapezoidal response and vice versa. This hypothesis was tested here, using computer-generated dynamic motion picture sequences representing a rectangle rotating about a horizontal axis.

\section{METHOD}

\section{Subjects}

A total of 115 subjects (four age groups) participated: 3-yearolds (median age $=3.5$ years, $\mathrm{N}=24$ ), 4 -year-olds (median age $=$ 4.3 years, $N=24$ ), 5 -year-olds (median age $=5.4$ years, $N=24$ ), and 21 -year-olds (median age $=21.0$ years, $\mathrm{N}=43$ ).

\section{Display}

Figure 1 shows the principles of polar projection and the generated sample patterns at different aspects of the slices of 

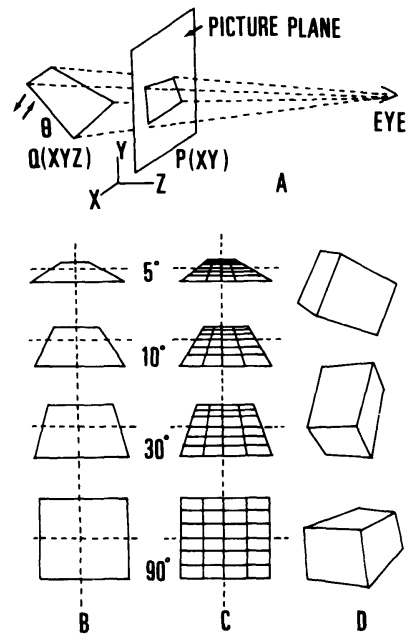

Figure 1. Dynamic-surface slant perception. Panel A: Principles of polar projection. The rotation of an imaging object in space $\left[Q_{(X, Y, Z}\right]$ can be plotted to the picture plane axes on a CRT [P(X,Y)] using matrix transformation. $\theta$ indicates the rotation angle along the $X$-axis. Panel B: Example slices of the computer-generated perspective views of a rectangle rotating along the $\mathrm{X}$-axis. Panel C: Views with a texture gradient density (linear perspective). Panel D: Example slices of an object rotating along different axes. Hidden lines were eliminated.

Table 1

Formula of Transformation Matrices for Rotation

\begin{tabular}{|c|c|c|c|}
\hline $\mathrm{QXYZ}_{\mathrm{X}(\alpha, \beta, \gamma)}=$ & $\mid \begin{array}{l}\cos \beta \cos \gamma \\
\sin \alpha \sin \beta \cos \gamma \\
-\cos \alpha \sin \gamma \\
\cos \alpha \sin \beta \cos \gamma \\
+\sin \alpha \sin \gamma\end{array}$ & $\begin{array}{l}\cos \beta \sin \gamma \\
\sin \alpha \sin \beta \sin \gamma \\
+\cos \alpha \cos \gamma \\
\cos \alpha \sin \beta \sin \gamma \\
-\sin \alpha \cos \alpha\end{array}$ & $\begin{array}{l}-\sin \beta \\
\sin \alpha \cos \beta \\
\cos \alpha \cos \beta\end{array}$ \\
\hline
\end{tabular}

dynamic rotation. When a surface is rotating about a horizontal axis, the angle of rotation can be derived from relative changes in the horizontal and vertical positions of projected points on the picture plane. This provides a potential cue of slant information (Braunstein, 1976). Figure 1 (Panel C) shows that as the slant of the surface increases, the rate of change of texture gradient increases. A mathematical description of this effect shows that gradient and slant are precisely related. This gradient cue enriches potential slant information, since a texture-density gradient is a sufficient stimulus for the perception of surface slant (Gibson, 1946). Consequently, if texture gradient information can be extracted by children, slant will be specified.

\section{Computational Procedures}

A random-scanning refresh-type CRT display (FACOM GD6233A with P5 phosphor) that has a laster resolution of 4,096 plotting positions on each axis was used. The line-drawing perspective figure was rotated at $60 \mathrm{deg} / \mathrm{sec}$. The formula of transformation matrices for rotation were as shown in Table 1 (Osaka, Note 1), where $\alpha, \beta$, and $\gamma$ indicate the angles of rotation along the $\mathrm{X}-, \mathrm{Y}-$, and $\mathrm{Z}$-axes, respectively. This calculation was performed by the computer graphics (FACOM 230/48 FORTRAN compiler). The rotating stimulus was observed at a $50-\mathrm{cm}$ viewing distance from the CRT. Subjects were asked to judge the perceived depth of nontextured (Panel B) and textured (Panel C) surfaces that were rotating about a horizontal axis.
They were also asked to report the perceived depth of the rotating "box" (Panel D). Children's verbal responses, such as "shrinkage" or "enlargement" of a figure, were classified as nonperceived depth responses (i.e., flat responses), and responses that indicated a "rotation" impression were classified as depth responses (Osaka, Note 1).

\section{RESULTS AND DISCUSSION}

Figure 2 shows the results. Effects of age $\left[\chi^{2}(3)=\right.$ $25.71, p<.01]$ and stimulus type $\left[\chi^{2}(2)=18.56\right.$, $\mathrm{p}<.01$ ] were found to be statistically significant. As age increases, percentage of "depth" responses increases. Stimulus Type D, however, was judged always to be perceived in depth for all age groups. Figure 2 indicates that if the texture gradient can be recognized, the slant of the surface is easily specified (Type C). However, rectangles displayed without texture tended to be judged as "flat" by children, but when they were viewed by adults (21-year-olds), the display created a continuous depth perception through rotation. Interestingly enough, Stimulus Type B did not elicit a depth response from 3-year-olds. This can be explained in terms of 3-year-olds' inability to specify the slant or inability to relate the slant with its shape. Several investigations of pictorial depth sensitivity in infants have demonstrated that responsiveness to pictorial depth information is absent in young infants (Bower, 1966; Day \& McKenzie, 1973). This is true for most of the 3-year-old children in the present study. In the last few years, however, it has been found that preschool children are sensitive to pictorial depth information. Benson and Yonas (1973) demonstrated that 3-year-olds were also quite sensitive to linear perspective information. However, about $75 \%$ of the 3 -year-olds failed to recognize the slant information in the present study. These results suggest that texture gradient can be associated with slant information after the age of 4 years and that shape-slant invariance is likely to develop as a function of age.

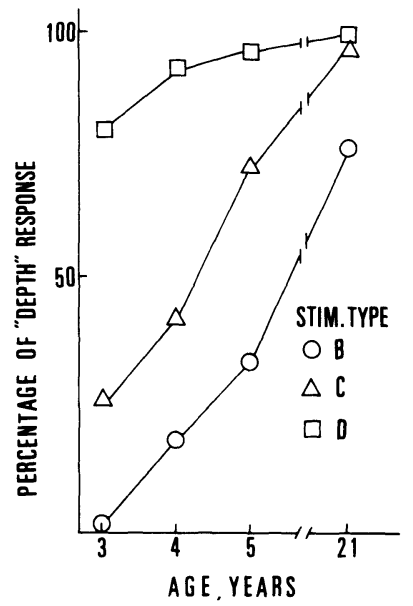

Figure 2. Percentage of "perceived depth" responses as a function of age in years. Parameter is stimulus type, as shown in Figure 1. 


\section{REFERENCE NOTE}

1. Osaka, N. Three-dimensional interactive computer graphics for perceptual experiments. Faculty of Letters Review, OtemonGakuin University, 1981, 15, 21-56. (in Japanese)

\section{REFERENCES}

Benson, C., \& Yonas, A. Development of sensitivity to static pictorial depth information. Perception \& Psychophysics, 1973, 13, 361-366.

Bower, T. G. R. Heterogeneous summation in human infants. Animal Behaviour, 1966, 14, 395-398.

Braunste in, M. L. Depth perception through motion. New York: Academic Press, 1976.

Day, R. H., \& McKenzie, B. E. Perceptual shape constancy in early infancy. Perception, 1973, 2, 315-320.
Gibson, J. J. The perception of the visual world. Boston: Houghton Mifflin, 1950.

Rosinski, R. R. The development of visual perception. Santa Monica, Calif: Goodyear, 1977.

Rosinski, R. R., \& Levine, N. P. Texture gradient effectiveness in the perception of surface slant. Journal of Experimental Child Psychology, 1976, 22, 261-271.

\section{NOTE}

1. Prior to the experimental session, the experimenter confirmed that each subject understood the meaning of "rotation" and "shrinkage." Stimuli were presented in random order for each subject.

(Received for publication December 21, 1982.) 